\title{
Primary testicular non-Hodgkin's lymphoma associated with hemophagocytic syndrome: Case report and review of the literature
}

\author{
WEIGANG REN ${ }^{1}$, ZHAOHUI ZHONG $^{1}$, XIAOKUN ZHAO ${ }^{1}$, JUNJIE YANG $^{2}$, LEI ZHANG ${ }^{1}$, \\ RAN XU ${ }^{1}$, SONGCHAO LI ${ }^{1}$, XUAN ZHU ${ }^{1}$, CHEN LU $^{3}$ and JOSEPH L. CHIN ${ }^{4}$ \\ Departments of ${ }^{1}$ Urology and ${ }^{2}$ Hematology, The Second Xiangya Hospital, Central South University, \\ Changsha, Hunan 410011; ${ }^{3}$ Department of Urology, Shanghai Changhai Hospital, Shanghai 200433, P.R. China; \\ ${ }^{4}$ Department of Urology and Oncology, University of Western Ontario, London, Ontario N6A 4V2, Canada
}

Received January 16, 2012; Accepted April 19, 2012

DOI: 10.3892/ol.2012.701

\begin{abstract}
Primary testicular non-Hodgkin's lymphoma (TNHL) was first described as a clinical entity in 1866. It is a rare disease which accounts for $1 \%$ of all non-Hodgkin's lymphoma, $2 \%$ of all extranodal lymphomas and $5 \%$ of all testicular neoplasms. Hemophagocytic syndrome (HPS) is a rare but distinct condition caused by inappropriate and dysregulated activation of the immune system. We report a 47-year-old man with primary TNHL who developed HPS four months after occurrence of scrotal swelling. To the best of our knowledge, primary TNHL associated with HPS has not previously been reported.
\end{abstract}

\section{Introduction}

Primary testicular non-Hodgkin's lymphoma (TNHL) was first described as a clinical entity in $1866(1,2)$. It is a rare disease which accounts for $1 \%$ of all non-Hodgkin's lymphoma (NHL) cases, $2 \%$ of all extranodal lymphomas and $5 \%$ of all testicular neoplasms (3). It is the most common type of testicular tumor in males between 60 and 80 years of age. TNHL is unique in its high incidence of bilateral involvement (8-38\%), and it is also the most common bilateral testicular tumor. TNHL has a predilection for spreading to non-contiguous extranodal sites, particularly the central nervous system (CNS) $(1,2,4-7)$. Hemophagocytic syndrome (HPS) also known as hemophagocytic lymphohistiocytosis (HLH) is characterized by the impaired or absent activity of natural killer (NK) cells and

Correspondence to: Dr Zhaohui Zhong, Department of Urology, The Second Xiangya Hospital, Central South University, Changsha, Hunan 410011, P.R. China

E-mail: jzhonguro@gmail.com

Key words: primary testicular non-Hodgkin's lymphoma, hemophagocytic syndrome, testicular natural killer/T-cell lymphoma, hemophagocytosis cytotoxic T-cells leading to cytokine dysregulation with proliferation and activation of histiocytes (8). HPS is characterized by fever, pancytopenia, liver dysfunction and hemophagocytosis in the bone marrow (9). HPS is divided into two types: primary familial and secondary. Secondary type is often associated with infection, autoimmune disease and malignancies.

In the present study, we report on a 47-year-old man with primary TNHL who developed HPS 4 months after occurrence of scrotal swelling. To the best of our knowledge, primary TNHL associated with HPS has not been previously reported.

The search engine PubMed was used to search the database Medline of the National Library of Medicine from 1966 to the present. The search engine Science Direct was used to search EMBASE from 1974 to November 2011. The search engine Web of Science was used to search the Science Citation Index from 1980 to November 2011. Earlier sources were obtained by cross referencing. Subject searches were conducted for hemophagocytosis, hemophagocytic syndrome, hemophagocytic lymphohistiocytosis, primary testicular non-Hodgkin's lymphoma and testicular natural killer/T-cell lymphoma. Studies considered to be of clinical importance and additional references of key articles were included.

The study was approved by the ethics committee of the university. Patient consent was also obtained.

\section{Case report}

A 47-year-old man presented with a 4-month history of bilateral scrotal swelling, prolonged fever, weakness and night sweats. The patient received antibiotics (trimethoprim and sulfamethoxazole) for suspected orchitis and was admitted to hospital due to pancytopenia and $15 \mathrm{~kg}$ weight loss.

Physical examination revealed a thin, chronically ill-appearing Chinese male. Vital signs were as follows: temperature, $37.7^{\circ} \mathrm{C}$; pulse, 95 beats per min; respiratory rate, 20 breaths per min and blood pressure, $85 / 60 \mathrm{mmHg}$. No scleral icterus was present. The patient's abdomen and the upper right arm had an approximately $2 \times 1 \mathrm{~cm}$ red rash, respectively, which faded when pressed. The oral cavity had good dentition without evidence of thrush. No neck masses 
or lymphadenopathies were palpable. The enlargement of the spleen was palpable under $6 \mathrm{~cm}$ of the left costal margin.

Hepatomegaly was not noted. No lower extremity edema was present. Neurological examination revealed normal cranial nerve function, reflexes, speech, mental status and gait. No focal weakness or sensory loss was present. Urological examination revealed bilateral hard testicular masses. The patient was otherwise in good health with no significant findings from medical history. Family history was non-contributory.

Blood routine examination revealed severe pancytopenia: hemoglobin was $93 \mathrm{~g} / \mathrm{dl}$, white blood cell count was $2.2 \times 10^{9} /$ liter and platelet count was $28 \times 10^{9} /$ liter. Routine urianlysis and stool routine examination were normal. Serum albumin was decreased to $27.5 \mathrm{~g} / 1$. Activated partial thromboplastin time was prolonged to $75.4 \mathrm{sec}$ and the concentration of fibrinogen was decreased to $0.55 \mathrm{~g} / \mathrm{l}$. Laboratory examinations on admission revealed highly elevated values of ferritin (957 ng/ml), lactate dehydrogenase (LDH; $1303 \mathrm{U} / \mathrm{l})$, serum aspartate aminotransferase (AST; $207 \mathrm{U} / \mathrm{l}$ ) and alanine aminotransferase (ALT; $82 \mathrm{U} / \mathrm{l}$ ), suggesting hematological malignancies, malignant tumor of fibrous connective tissue and severe infection. Therefore, an extensive rheumatologic and infectious investigation was performed during hospitalization. Rheumatologic work-up, including antinuclear antibodies, anti-double-stranded DNA antibodies, complement $\mathrm{C} 3$ and $\mathrm{C} 4$ levels, antimitochondrial antibodies, antiscleroderma-70 antibodies, anti-Ro/SS-A and anti-La/SS-B antibodies, were all within normal limits.

Infectious work-up included Mycoplasma pneumoniae $\mathrm{IgG}$ and IgM levels, Herpes simplex virus IgG and IgM levels, Toxoplasma gondii $\operatorname{IgG}$ and IgM levels, rubella IgG and IgM levels, cytomegalovirus IgG and IgM levels, Epstein-Barr virus antibodies and an hepatitis profile. Results from the infectious disease laboratories were within normal limits. Other laboratory tests performed, including multiple blood cultures, international normalized ratio, erythrocyte sedimentation rate and immunoglobulin levels (IgA, $\operatorname{IgM}, \operatorname{IgG}$ and $\operatorname{IgE}$ ), were also within normal limits. Antibodies to HIV1/2 and antibody to Treponema pallidum were negative. The $\beta$ human chorionic gonadotropin (HCG) and $\alpha$-fetoprotein (AFP) levels were within normal limits.

Ultrasonography revealed bilateral testicular enlargement (56 cc right testis, $36 \mathrm{cc}$ left testis) and multifocal, intensely hypoechoic areas, which demonstrated enhanced flow velocity following Color Doppler sonography. A computed tomography scan of the chest, abdomen and pelvis demonstrated lung infection of the left inferior and right middle lobe, adiposis hepatica, splenomegaly and bilateral testicular enlargement; however, no lymph node enlargement was found (Fig. 1).

Contrast-enhanced MRI of the brain revealed no abnormalities except for paranasal sinusitis. A bone marrow biopsy and aspiration were performed. The biopsy results demonstrated the following: bone marrow active proliferation; reduction in the cellularity in all lineages; granulocytic series accounting for $50 \%$, with relatively normal morphology; erythrocytic series accounting for $30 \%$, with intermediate erythroblasts and largely acidophilic normoblasts, but no abnormal morphology; lymphocytic series accounting for 17\%; megakaryocyte with normal distribution, scattered piles of platelets; large platelets and hemophagocytosis (Fig. 2).

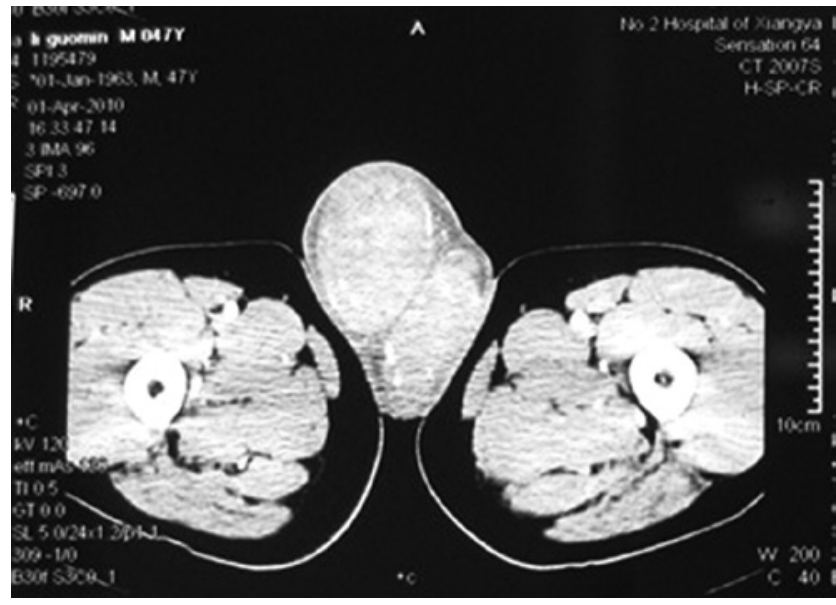

Figure 1. A computed tomography scan of the pelvis demonstrated bilateral testicular enlargement.

According to the presented symptoms, laboratory examinations and a bone marrow cell examination, the patient was diagnosed with HPS. No other hereditary or acquired diseases related to HPS were found. As the severe pancytopenia persisted, regular substitution of erythrocytes, $\gamma$ globulins, albumin and platelets was required. Since the bilateral testicular tumor was the only lesion potentially leading to HPS, a bilateral orchiectomy was performed. Histological sections revealed small to medium-sized neoplastic cells, typical heteromorphism and prominent necrosis infiltrating and destroying seminiferous tubules (Fig. 3). The immunohistochemical staining of paraffin-embedded sections demonstrated reactivity for LCA, Vim, CD45RO, TIA-1, CD43, CD56 focal, $\mathrm{IgG}$ and $\mathrm{Ki}-67$. The results of staining for CD10, CD79a, CD3, CD5, CD1a, CD30, CD138, CD38, CD20, TdT, MoPo, Mum-1, Ck, EMA and PLAP were negative. Staining for CD45RB and IgA was positive (Fig. 4). The diagnosis was bilateral testicular non-Hodgkin's malignant lymphoma, as with NK/T-cell lymphoma.

Despite bilateral orchiectomy, the patient's clinical condition rapidly deteriorated with persistent fever and severe pancytopenia. Given the extremely poor prognosis, the patient refused chemotherapy and was discharged and transferred to the local hospital, where the patient's clinical condition deteriorated with palliative supportive care. The patient succumbed to acute multiple organ failure 2 weeks later.

\section{Discussion}

To the best of our knowledge, this is the first report of a case in which HPS was caused by a primary testicular non-Hodgkin's malignant lymphoma, as with NK/T-cell lymphoma. TNHL has a predilection for dissemination to non-contiguous extranodal sites, including the CNS, Waldeyer's ring, skin and lungs $(1,5,10,11)$. According to the Working Formulation of the United States National Cancer Institute, approximately 68\% of TNHL cases are classified as intermediate grade, diffuse large B-cell subtype, followed by high-grade, diffuse small non-cleaved subtype in approximately $30 \%$ of patients $(1,11)$. There is no prognostic advantage for any pathological subtype (11). Immunohistochemistry (IHC) studies confirm the 


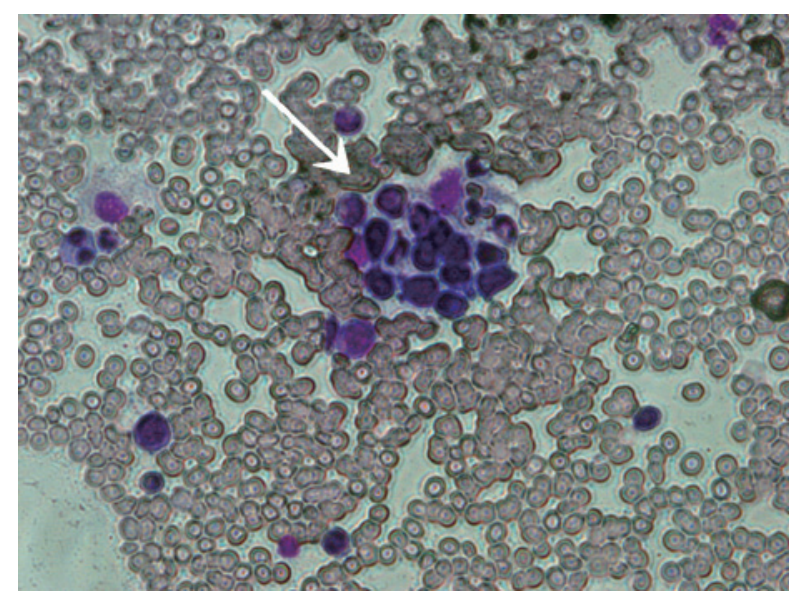

Figure 2. Bone marrow examination revealed a reduction in the cellularity in all lineages. Macrophages were increased with signs of hemophagocytosis. The white arrow indicates erythrocytes within the cytoplasm of a macrophage.

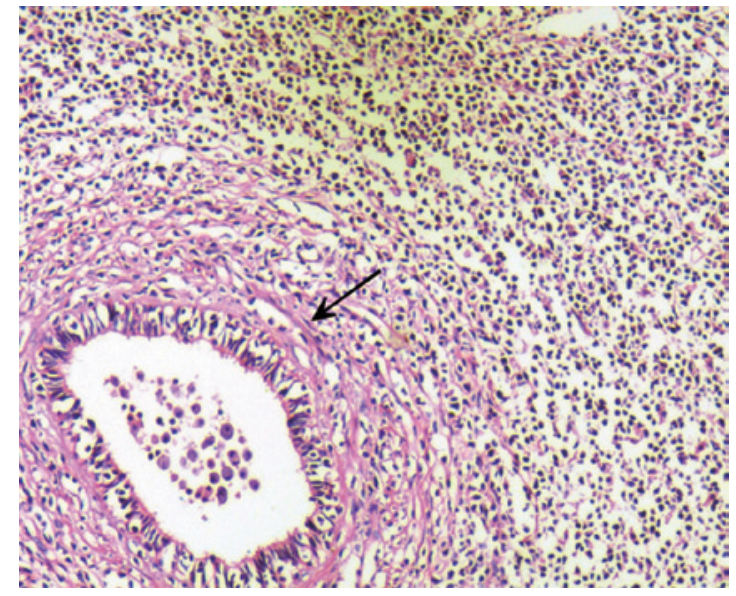

Figure 3. Neoplastic cells were small to medium-sized, with typical heteromorphism and prominent necrosis infiltrating and destroying seminiferous tubules. Arrow indicates area of seminiferous tubules (hematoxylin and eosin staining; original magnification, $\mathrm{x} 400)$.
A

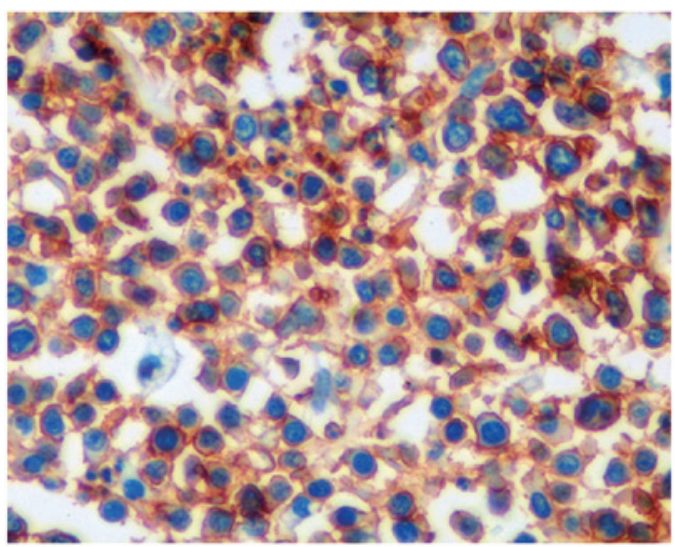

C

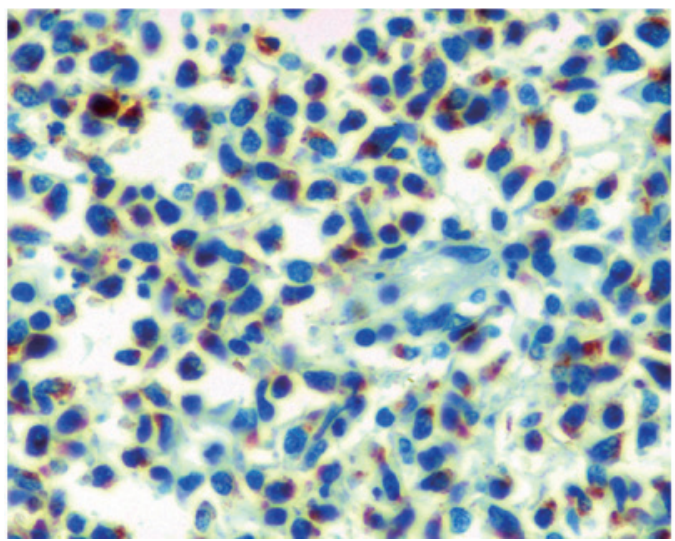

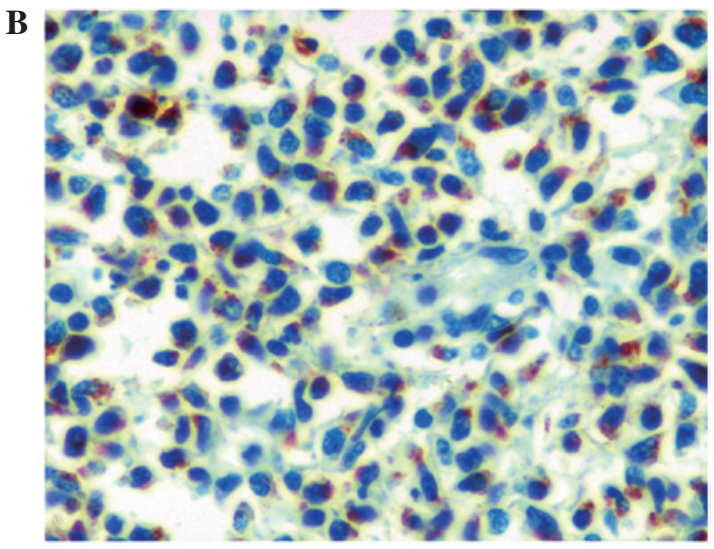

D

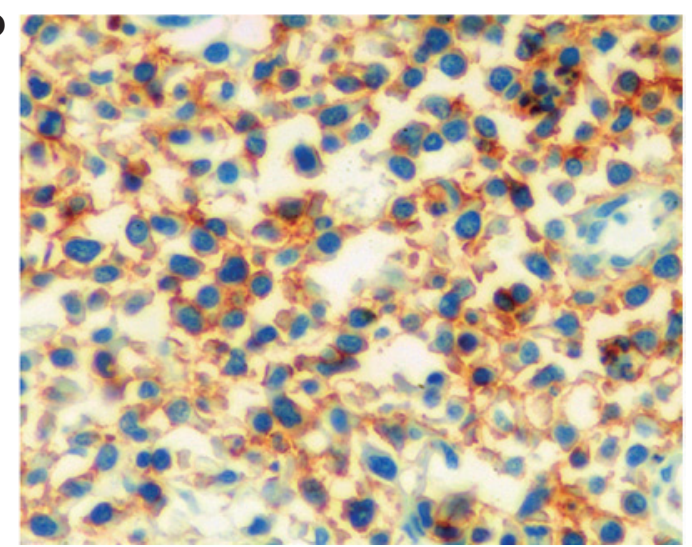

Figure 4. Immunohistochemical stain demonstrating (A) LCA-positive tumor cells completely surrounding and infiltrating seminiferous tubule, (B) TIA-1-positive tumor cells completely filling the entire field of vision, (C) CD43-positive tumor cells completely filling the entire field of vision and (D) CD45RO-positive tumor cells completely filling the entire field of vision. (A and D) Original magnification, x100. (B and C) Original magnification, x400.

majority of TNHL cases to be of B-cell origin, with less occurrence of T-cell lymphoma (1,12).

Extra-nasal NK/T-cell lymphomas, including the testicular lymphoma detected in our patient, are rare. The most common NK/T-cell lymphomas are nasal or nasal-type. Although primary extranasal tumors occur, testicular lesions are particularly uncommon, whether as primary tumors or as part of multifocal disease presentation. NK/T-cell lymphomas with testicular involvement share a number of clinicopathological features with NK/T-cell lymphomas of other extranasal sites, including a tendency to occur in individuals of Asian, Mexican and South American origin and a notably poor clinical prog- 
nosis $(13,14)$. Almost all patients whose lesions appeared to be limited to the testes relapsed within 6 months and succumbed to widespread dissemination of the skin, CNS, gastrointestinal (GI) tract or lung within 1 year, regardless of treatment $(15,16)$. Ornstein et al demonstrated that in contrast to testicular B-cell lymphoma, primary testicular NK/T-cell lymphoma occurred in younger males; the average age at presentation for a solitary testicular lesion was 44 years, and $60 \%$ of cases were diagnosed in patients younger than 40 years (17). In our case report, the patient also had a rapidly progressive, fatal course, consistent with findings from previous studies.

Concurrent occurrence or association with HPS may be part of the reason for the fulminant course. Viral infection is the most frequent trigger of secondary HPS. Malignancy is one of the major causes of HPS, and lymphoproliferative disease is the main type of malignancy associated with HPS (18). T-cell or B-cell lymphoma with HPS can occur in immunocompromised patients, including individuals with HIV infection and recipients of organ or bone marrow transplants, as well as in otherwise immunocompetent populations (19). Notably, HPS is frequently reported among patients who present with virusinduced lymphoproliferative disorders, including EBV-driven B-cell lymphoma or human Herpes virus-8-associated disease (20). HPS is, therefore, able to complicate the course of T-cell lymphoma, Hodgkin's disease, NK-cell leukemia, myeloproliferative disorders and acute leukemia (21). HPS is a highly fatal disease if untreated. Since HPS is rare, no randomized controlled clinical trials examining potential treatments have been conducted. The immediate aim of therapy is suppression of the increased inflammatory response and control of cell proliferation using immunosuppressive or immunomodulatory agents and cytotoxic drugs. Urologists require awareness of the occurrence of HPS in testicular tumor patients with persistent fever, organomegaly and cytopenia. Uropathologists should be aware of this rare entity which may only be diagnosed after extensive immunohistochemical studies.

To obtain a pathological diagnosis, bone marrow cell examination and orchiectomy should be performed as soon as possible. Management of HPS relies on early diagnosis, identification of a triggering pathogen or an underlying disease, and control of the lymphocyte/macrophage proliferation and activation. Specific antimicrobial therapy can be beneficial in selected cases. Severe cases are treated with chemotherapy, generally an etoposide-containing regimen, and bone marrow transplantation is the treatment for familial, severe and persistent non-familial cases.

\section{References}

1. Shahab N and Doll DC: Testicular lymphoma. Semin Oncol 26: 259-269, 1999.
2. Ballen KK and Hasserjian RP: Case records of the Massachusetts General Hospital. Weekly clinicopathological exercises. Case 15-2004. A 31-year-old man with bilateral testicular enlargement. N Engl J Med 350: 2081-2087, 2004

3. Touroutoglou N, Dimopoulos MA, Younes A, et al: Testicular lymphoma: late relapses and poor outcome despite doxorubicin-based therapy. J Clin Oncol 13: 1361-1367, 1995.

4. Eskey CJ, Whitman GJ and Chew FS: Malignant lymphoma of the testis. AJR Am J Roentgenol 169: 822, 1997.

5. Buskirk SJ, Evans RG, Banks PM, et al: Primary lymphoma of the testis. Int J Radiat Oncol Biol Phys 8: 1699-1703, 1982.

6. Lobo FD, Bansal R, Naik R, et al: Primary testicular lymphoma. J Indian Med Assoc 96: 193-194, 1998.

7. Sussman EB, Hajdu SI, Lieberman PH and Whitmore WF: Malignant lymphoma of the testis: a clinicopathologic study of 37 cases. J Urol 118: 1004-1007, 1977.

8. Rouphael NG, Talati NJ, Vaughan C, et al: Infections associated with haemophagocytic syndrome. Lancet Infect Dis 7: 814-822, 2007.

9. Janka GE: Familial and acquired hemophagocytic lymphohistocytosis. Eur J Pediatr 166: 95-109, 2007.

10. Lagrange JL, Ramaioli A, Theodore CH, et al: Non-Hodgkin's lymphoma of the testis: a retrospective study of 84 patients treated in the French anticancer centres. Ann Oncol 12: 1313-1319, 2001.

11. Tepperman BS, Gospodarowicz MK, Bush RS and Brown TC: Non-Hodgkin lymphoma of the testis. Radiology 142: 203-208, 1982 .

12. Moller MB, D'Amore F and Christensen BE: Testicular lymphoma: a population-based study of incidence, clinicopathological correlations and prognosis. The Danish Lymphoma Study Group, LYFO. Eur J Cancer 30A: 1760-1764, 1994.

13. Ko Y, Cho E-Y, Kim J-E, et al: NK and NK-like T-cell lymphoma in extranasal sites: a comparative clinicopathological study according to site and EBV status. Histopathology 44: 480-489, 2004.

14. Chan J, Sin V, Wong K, et al: Nonnasal lymphoma expressing the natural killer cell marker CD56: a clinicopathologic study of 49 cases of an uncommon aggressive neoplasm. Blood 89: 4501-4513, 1997.

15. Cheung MM, Chan JK, Lau WH, et al: Primary non-Hodgkin's lymphoma of the nose and nasopharynx: clinical features, tumor immunophenotype, and treatment outcome in 113 patients. J Clin Oncol 16: 70-77, 1998 .

16. Falini B, Pileri S, De Solas I, et al: Peripheral T-cell lymphoma associated with hemophagocytic syndrome. Blood 75: 434-444, 1990.

17. Ornstein DL, Bifulco CB, Braddock DT and Howe JG: Histopathologic and molecular aspects of $\mathrm{CD}^{+} 6^{+}$natural killer/ T-cell lymphoma of the testis. Int J Surg Pathol 16: 291-300, 2008.

18. Janka GE: Hemophagocytic syndromes. Blood Rev 21: 245-253, 2007.

19. Han AR, Lee HR, Park BB, et al: Lymphoma-associated hemophagocytic syndrome: clinical features and treatment outcome. Ann Hematol 86: 493-498, 2007.

20. Fardet L, Blum L, Kerob D, et al: HHV-8 associated hemophagocytic lymphohistiocytosis in HIV-infected patients. Clin Infect Dis 37: 285-291, 2003.

21. Chuang HC, Lay JD, Chuang SE, et al: EBV latent membrane protein-1 down-regulates TNF-[alpha] receptor-1 and confers resistance to $\mathrm{TNF}$-[alpha]-induced apoptosis in $\mathrm{T}$ cells: implication for the progression to T-cell lymphoma in EBV-associated hemophagocytic syndrome. Am J Pathol 170: 1607-1617, 2007. 